OPEN ACCESS

Edited by:

Hans Van Rostenberghe,

Universiti Sains Malaysia

(USM), Malaysia

Reviewed by:

Naeem Mubarak

Lahore Medical and Dental

College, Pakistan

Scott Weissman,

Seattle Children's Hospital,

United States

*Correspondence:

Philip Lee

phle@montefiore.org

Specialty section:

This article was submitted to

Pediatric Infectious Diseases,

a section of the journal

Frontiers in Pediatrics

Received: 03 March 2021

Accepted: 16 July 2021

Published: 16 August 2021

Citation:

Lee P, Kim M, Herold BC and Soma VL (2021) Under-utilization of Narrow-Spectrum Antibiotics in the Ambulatory Management of Pediatric UTI: A Single-Center Experience.

Front. Pediatr. 9:675759

doi: 10.3389/fped.2021.675759

\section{Under-utilization of} Narrow-Spectrum Antibiotics in the Ambulatory Management of Pediatric UTI: A Single-Center Experience

\author{
Philip Lee ${ }^{1,2 *}$, Mimi Kim ${ }^{3}$, Betsy C. Herold ${ }^{1}$ and Vijaya L. Soma ${ }^{1}$ \\ ${ }^{1}$ Division of Pediatric Infectious Disease, The Children's Hospital at Montefiore, Bronx, NY, United States, ${ }^{2}$ Department of \\ Pharmacy, The Children's Hospital at Montefiore, Bronx, NY, United States, ${ }^{3}$ Division of Biostatistics, Department of \\ Epidemiology \& Population Health, Albert Einstein College of Medicine, New York City, NY, United States
}

Objective: There are urinary tract infection (UTI) guidelines for treatment of patients $<2$ years old, but there is a paucity of data for other pediatric age groups including the potential role for stewardship to reduce prescription of broad-spectrum antibiotics. We assessed practice patterns for the diagnosis and empiric treatment of UTI for outpatient and school health sites affiliated with a large urban pediatric medical center. We hypothesized that outpatient providers under-utilize narrow-spectrum antibiotics, such as first-generation cephalosporins, for uncomplicated UTI.

Study Design: Retrospective study from December 1st, 2015 to May 31st, 2016.

Results: The study population included 903 children (70.1\% female) with a median age of 11 years, evaluated in an outpatient clinic ( $n=780,86.4 \%)$ or school health site $(n=123,13.6 \%)$. E. coli was the most common urinary pathogen $(50.9 \%)$ and $92.6 \%$ of $E$. coli isolates were susceptible to cephalexin. However, cephalexin was prescribed empirically for only $12.8 \%$ of patients. In contrast, sulfamethoxazole-trimethoprim was commonly prescribed, but only $79 \%$ of $E$. coli isolates were susceptible. Antibiotics were discontinued in only three of 48 children who had negative urine cultures.

Conclusions: Cephalexin may be the most appropriate first-line choice for management of outpatient UTI for our patient population. Antibiotics were rarely discontinued for those with negative urine cultures. Antibiotic stewardship in the outpatient setting could reduce unnecessary antibiotic exposure in the management of pediatric UTI.

Keywords: urinary tract infection, pediatric, antibiotic, outpatient, school health

\section{INTRODUCTION}

Urinary tract infection (UTI) is a common outpatient pediatric diagnosis. There are guidelines for the diagnosis and management of uncomplicated UTI in children 2-24 months of age but not for older children and adolescents $(1,2)$. The gold standard for diagnosis is bacterial culture with antibiotic selection based on susceptibility testing. However, results of these diagnostic tests are not immediately available and thus clinicians face the dilemma of whether to empirically treat for a presumptive UTI or delay treatment in a symptomatic patient. Some advocate using urinary nitrites and/or leukocyte esterase on a dipstick to guide decisions about whether to start empiric 
therapy, but the sensitivity and specificity of these rapid pointof-care tests are variable (2-5). Similarly, quantification of white blood cells and bacteria by microscopy is rarely available in outpatient settings and the sensitivity and specificity of microscopy ranges from 50-96\% (2-5).

If a clinician elects to start antibiotic treatment prior to having culture and susceptibility results, empiric therapy is usually based on community patterns of bacterial resistance $(1,6,7)$. Mathematical modeling studies suggest that drugs such as amoxicillin, sulfamethoxazole-trimethoprim (SXT) and ciprofloxacin should not be prescribed for empiric therapy if local resistance rates for common urinary pathogens such as Escherichia (E.) coli exceed $20 \%(8,9)$. E. coli rates of resistance in developed nations have been reported as high as 50 and $30 \%$ for SXT and amoxicillin, respectively (10). Studies in adults have shown that treatment of community-acquired $E$. coli UTIs with either SXT or ciprofloxacin is associated with recurrent UTIs and development of resistance within 6-12 months of initial presentation $(11,12)$. However, similar data in pediatrics is limited.

Given the paucity of data in children, particularly those over 2 years of age, we conducted a retrospective study to assess the practice patterns for diagnosis and empiric treatment of pediatric UTI in a large urban outpatient network consisting of traditional ambulatory sites and school-based health centers. Urine culture results and susceptibility patterns were reviewed. We hypothesized that outpatient providers under-utilize narrowspectrum antibiotics, defined here as penicillins and firstgeneration cephalosporins, compared to other, broader-spectrum antibiotics for uncomplicated UTI $(13,14)$.

\section{METHODS}

\section{Study Design}

A retrospective study of patients $>2$ years and under 21 years of age who sought care in one of 54 outpatient sites associated with The Children's Hospital at Montefiore, Bronx, NY between December 2016 and June 2017 and had an ICD-9 code associated with UTI at the visit (ICD-9 codes 599, 791.9, 788.1, 788.41, 788.63) was conducted. Sites included 17 school health programs and 37 pediatric clinics. Demographics, clinical symptoms, urine testing results, prescribed antibiotics, and related return visits within 6 months of the initial encounter were extracted from the electronic medical record (EMR). Patients were excluded if they were treated with an antimicrobial for a condition other than UTI, were pregnant, had congenital genitourinary abnormalities, or had a past positive urine culture since birth recorded in the EMR. Institutional Review Board approval for the study was obtained from the Albert Einstein College of Medicine Office of Human Research Affairs.

\section{Definitions}

A positive urine screen was defined as a dipstick with detectable leukocyte esterase or nitrites or urine with microscopic findings

Abbreviations: SXT, Sulfamethoxazole-trimethoprim; UTI, urinary tract infection; UA, urinalysis. of $\geq 10$ white blood cells (WBC) per high-powered-field (HPF). All culture and susceptibility testing were performed by the Montefiore Clinical Microbiology Lab. A positive urine culture was defined as having $\geq 100,000$ colony-forming units $(\mathrm{CFU}) / \mathrm{mL}$ of a single bacterium from a cleanly voided specimen. If two or more organisms were present, the culture was included only if one was present at $\geq 100,000 \mathrm{CFU} / \mathrm{mL}$ and the other was $<10,000 \mathrm{CFU} / \mathrm{mL}$. Testing was performed using the Phoenix 100 instrument with automated dilution and software version $6.35 \mathrm{~A}$ (Becton, Dickinson and Company, Franklin Lakes, NJ) by the Montefiore Medical Center Microbiology Department. Return visits to a clinic were categorized as those occurring within 6 months from the initial visit for a new UTI symptom.

\section{Statistical Analyses}

Positive predictive value (PPV) was defined as proportion of true cases (based on positive urine culture) among all participants with a positive screening test and negative predictive value (NPV) was defined as true non-cases (negative urine culture) among all participants with a negative screening test. Chi square of independence and Fisher's exact tests were used to compare groups with respect to binary outcomes. Continuous variables were compared using Student $t$-test. The Kaplan-Meier method and log rank test were used to estimate and compare between antibiotic groups the distribution of time to clinic return for recurrent UTI symptoms. Descriptive statistics were used when comparing demographics. A two-sided $p$-value of $<0.05$ was considered statistically significant. All analyses were performed using GraphPad and SAS.

\section{RESULTS}

There were 1,199 patients with an ICD-9 code associated with UTI over the study period and 1,138 had a retrievable EMR. The following patients were excluded: 33 that were treated with an antimicrobial for other conditions (including 22 diagnosed

TABLE 1 | Subject demographics.

\# Patients $(n=903)$

Sex, $n(\%)$
Male
Female
Race, $n(\%)$
American Indian or Alaska Native
Asian
Black
White
Hispanic
Other
Declined to Answer
Median age in years, ( \pm SD)
Outpatient Sites
Clinical, $n$ (\%)
School Health, $n(\%)$

$270(29.9 \%)$

$633(70.1 \%)$

$7(0.8 \%)$

$15(1.7 \%)$

$259(28.7 \%)$

$67(7.4 \%)$

$350(38.8 \%)$

$77(8.5 \%)$

$128(14.2 \%)$

$11 \pm 5.9$

$780(86.4 \%)$

$123(13.6 \%)$ 


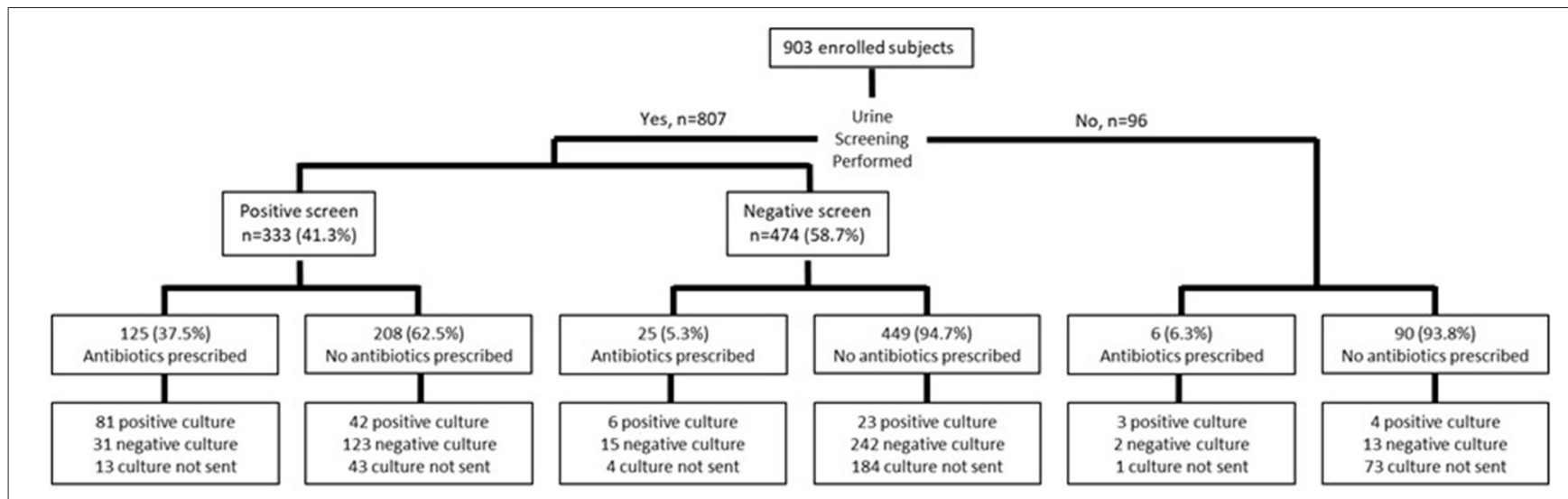

FIGURE 1 | Testing and empiric treatment in pediatric outpatients diagnosed with UTI.

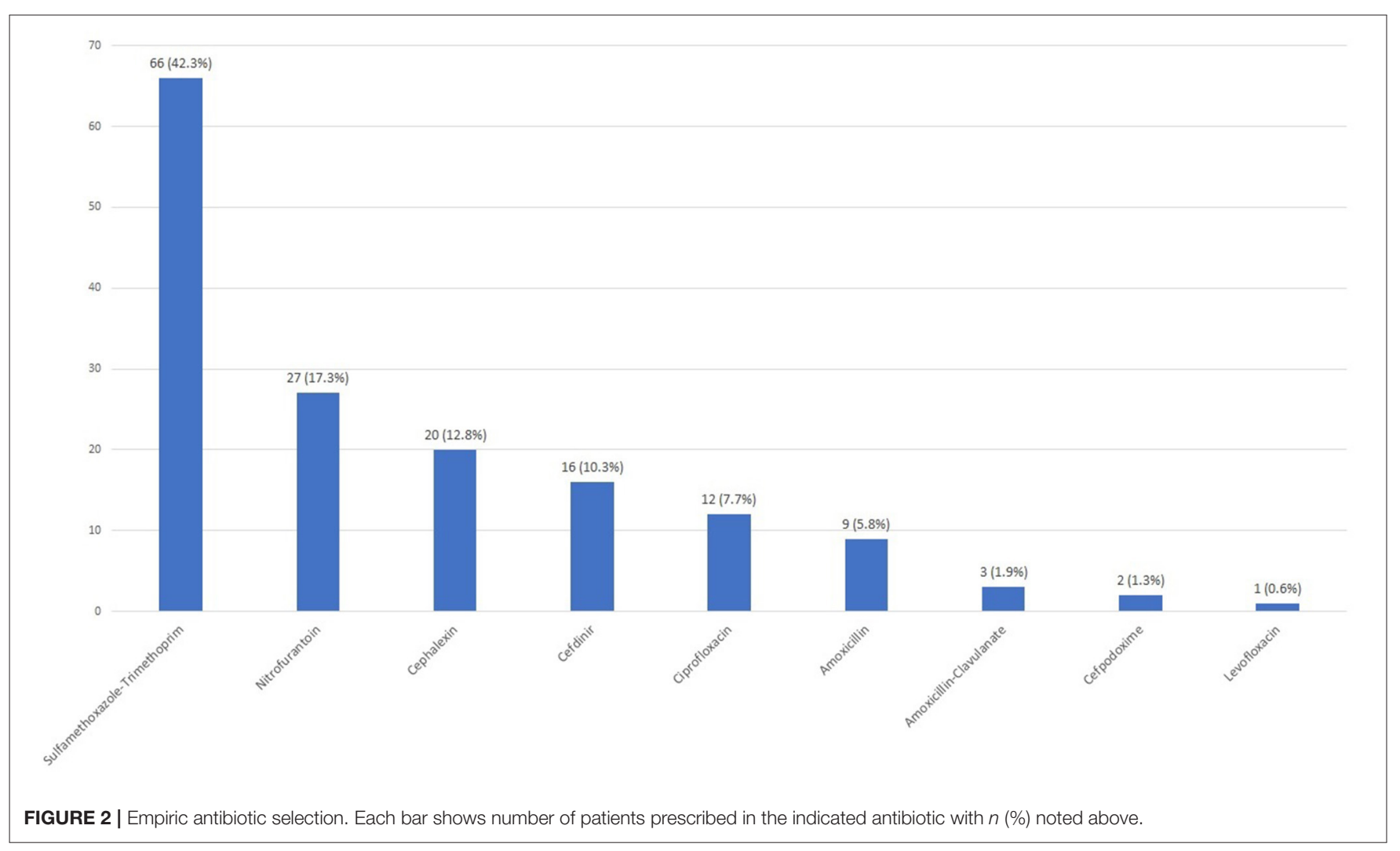

with sexually transmitted infection or fungal vulvovaginitis), 21 who were $\leq 2$ years old, and 181 patients with a previous positive urine culture, yielding a final study population of 903 patients. The majority $(n=780,86.4 \%)$ were seen at an outpatient clinic and $123(13.6 \%)$ were evaluated at a school health clinic. The median age of the overall study cohort was 11 years (SD: \pm 5.9 , range 2-21 years). The patients from school health clinics were significantly older than those from outpatient clinics, $16 \pm 3$ (median \pm SD) vs. $9 \pm 6$ years, respectively $(p<0.001)$. Other demographic characteristic detailed in
Table 1 reflect the community population with respect to race and ethnicity.

A urine screen was performed in $89.4 \%(n=807)$ of patients; 473 had a point of care dipstick, 129 had urine microscopy ordered and performed in the microbiology laboratory and 205 had both screening tests (Figure 1). Urine cultures were obtained in a majority of patients who had a screening test performed $(563 / 807,69.8 \%)$, but were more likely to be sent in those with a positive compared to a negative screen $(277 / 333,83.1 \%$ vs. $286 / 474,60.3 \%, p<0.001)$. A culture was also sent in $22 / 96$ 
(22.9\%) of those who had no screening test performed. The urine culture was positive in $123 / 277(36.9 \%)$ with a positive urine screen (PPV: 44.4\%, 95\% CI: 40.8\%, 48.1\%), and 30/286 (6.3\%) with a negative screen (NPV: $89.5 \%, 95 \%$ CI: 86\%, 92.2\%). Among those who had no screening test performed, 7/96 (4.3\%) had a positive urine culture.

Empiric antibiotics were prescribed more frequently in those with a positive screen $(124 / 333,37.2 \%)$ compared to those with a negative screen $(25 / 474,2.3 \%)(p<0.001)$ (Figure 1). The most commonly prescribed empiric antibiotics were SXT (42.3\%), nitrofurantoin (17.3\%), cephalexin $(12.8 \%)$ and ciprofloxacin (7.7\%) (Figure 2). School health providers prescribed SXT at a higher rate $(65.5 \%)$ than other outpatient clinicians $(37 \%), p<$ 0.01 (Table 2).

Urine cultures were sent in 127 of the 159 (79.9\%) patients who were prescribed empiric antibiotics; 79 (62.2\%) of the cultures were positive (Figure 3). E. coli was the most common pathogen identified and accounted for $50.9 \%$ of positive cultures (Figure 4). Other isolates identified included mixed cultures

TABLE 2 | Empiric antibiotic choice, by outpatient site, $n$ (\%).

\begin{tabular}{lccc}
\hline Antibiotic & $\begin{array}{c}\text { School health } \\
(\mathbf{N = 2 9 )}\end{array}$ & $\begin{array}{c}\text { Outpatient } \\
(\mathbf{N = 1 2 7 )}\end{array}$ & $\boldsymbol{p}$-value \\
\hline Cephalexin & $1(3.4 \%)$ & $19(15 \%)$ & 0.1262 \\
Sulfamethoxazole-trimethoprim & $19(65.5 \%)$ & $47(37 \%)$ & 0.0066 \\
Nitrofurantoin & $6(20.7 \%)$ & $21(16.5 \%)$ & 0.5918 \\
Ciprofloxacin & $2(6.9 \%)$ & $10(7.9 \%)$ & 1 \\
Amoxicillin & $1(3.4 \%)$ & $8(6.3 \%)$ & 1 \\
Other & 0 & $22(17.3 \%)$ & -
\end{tabular}

(25.2\%), various gram-positive bacteria (14.5\%), Proteus mirabilis (3.6\%), and Klebsiella pneumoniae (1.9\%).

Based on 2016 Clinical and Laboratory Standards Institute (CLSI) breakpoints (13), rates of E. coli susceptibility were highest for cephalexin $(92.6 \%)$, nitrofurantoin $(98.8 \%)$ and ciprofloxacin (93.8\%) and lowest for SXT (79.8\%), tetracycline (76.2\%), ampicillin-sulbactam (64.3\%) and ampicillin (61.9\%). Only $2.5 \%$ of $E$. coli isolates produced extended-spectrum beta lactamases (ESBL) based on ceftriaxone susceptibility (13) (Table 3).

There were seven cases in which the bacteria isolated was not susceptible to the empiric antibiotic prescribed and the antibiotic was appropriately changed in all but one case. Complete discontinuation of the empiric antibiotic in the setting of a negative urine culture, however, was infrequent. There were 48 patients in whom urine cultures yielded no growth, but 45 (93.8\%) continued therapy; only three had treatment discontinued. Two patients who were continued on therapy were prescribed a different antibiotic because of persistent symptoms (Figure 3).

Treatment with cephalexin was associated with the lowest rate of clinic return for recurrent or persistent symptoms within 6 months of initial presentation, but this did not differ significantly compared to other antibiotics as shown in the Kaplan-Meier plot (Figure 5). Return rates were 15\% (3/20) for cephalexin, 19.7\% $(13 / 66)$ for SXT, $18.5 \%$ (5/27) for nitrofurantoin and 16.7\% (2/12) for ciprofloxacin. There were no returns within the 1st month when patients were treated with cephalexin or ciprofloxacin.

\section{DISCUSSION}

We hypothesized that outpatient pediatric providers underutilize narrow-spectrum antibiotics for uncomplicated UTI and

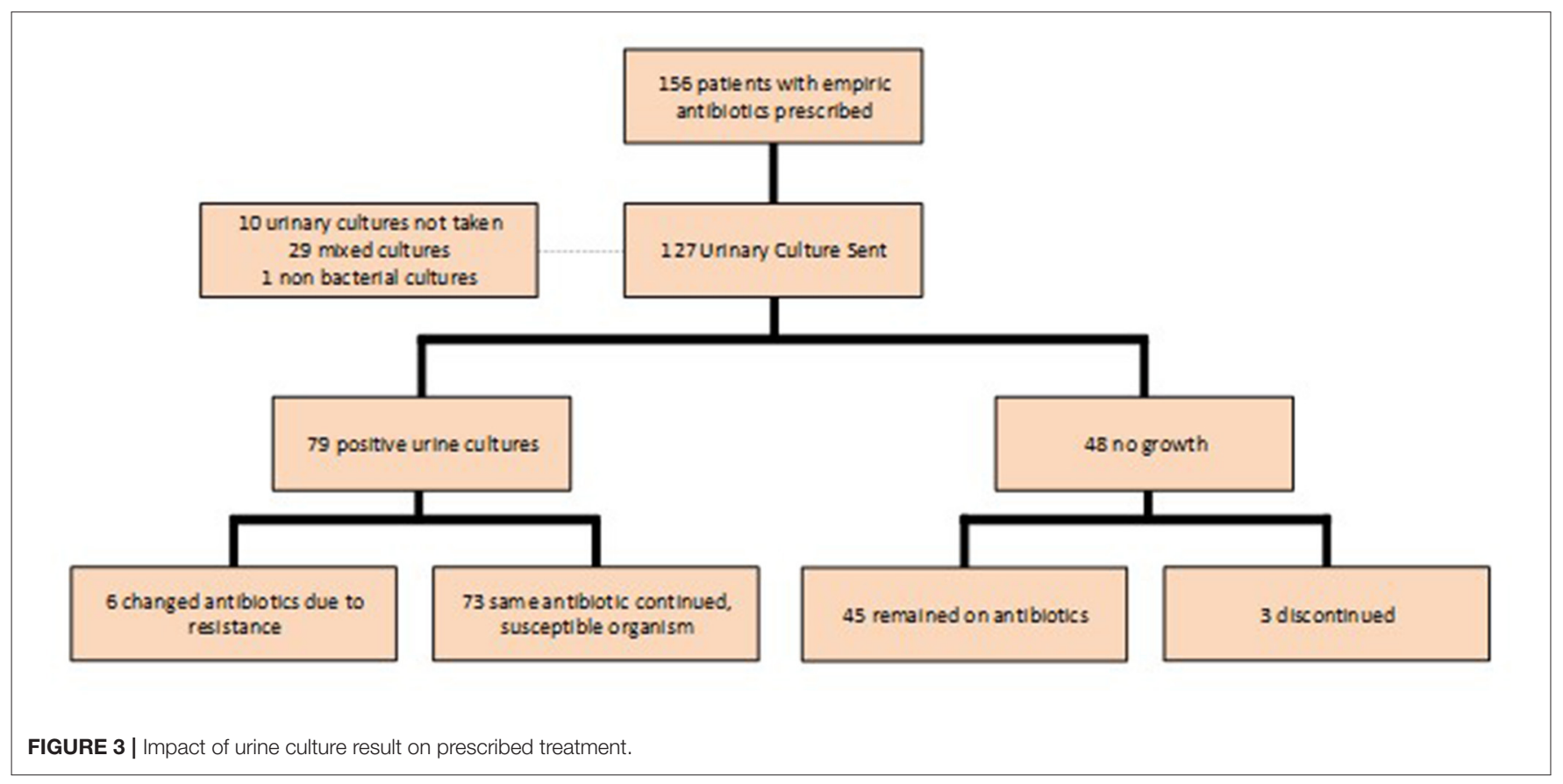




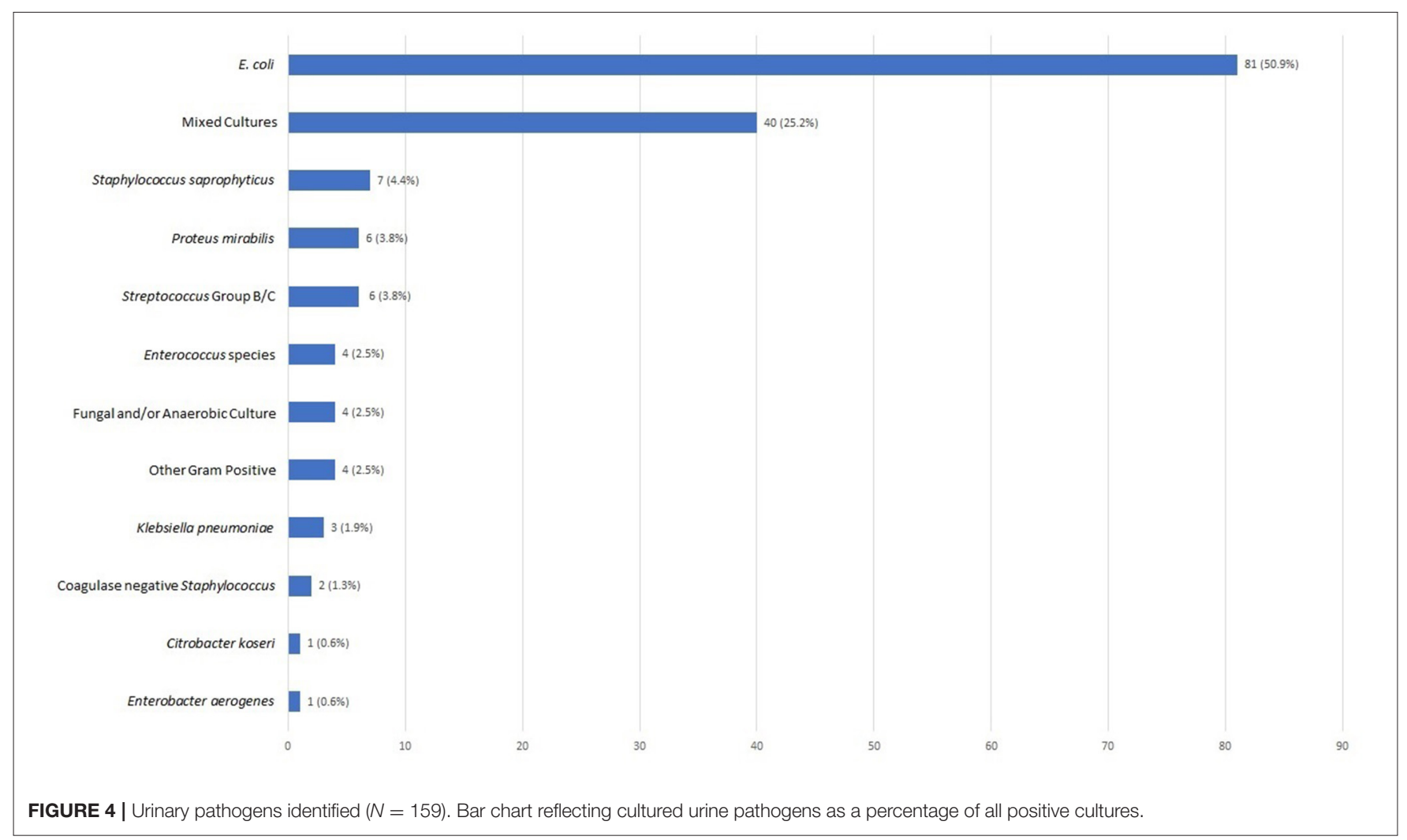

TABLE 3 | E. coli susceptibility to antibiotics across all age groups.

\begin{tabular}{lc}
\hline & $\begin{array}{c}\text { Number of susceptible } \\
\text { isolates (Total } \mathbf{N = 8 1 )}\end{array}$ \\
\hline Cefazolin $^{\dagger}$ & $75(92.6 \%)$ \\
SXT & $64(79 \%)$ \\
Ampicillin & $51(63 \%)$ \\
Ampicillin-Sulbactam & $52(64.2 \%)$ \\
Ceftriaxone & $79(97.5 \%)$ \\
Ciprofloxacin & $76(93.8 \%)$ \\
Gentamicin & $72(88.9 \%)$ \\
Nitrofurantoin & $80(98.8 \%)$ \\
Tetracycline & $61(75.3 \%)$ \\
\hline
\end{tabular}

${ }^{\dagger}$ Susceptibility refers to the new cefazolin breakpoint to treat E. coli, K. pneumoniae and P. mirabilis UTIs.

found this to be true of outpatient practices affiliated with our institution. A high percentage of the E. coli isolates were susceptible to cephalexin, but this antibiotic was infrequently prescribed as the empiric choice in both the school health and outpatient clinic setting. Cephalexin was the least prescribed in the school health setting and the third least in the outpatient setting. Physicians prescribed SXT or nitrofurantoin most often. Moreover, even after organism identification and susceptibility results were available, treatment was only changed if the organism was resistant to the initial antibiotic; narrowing of

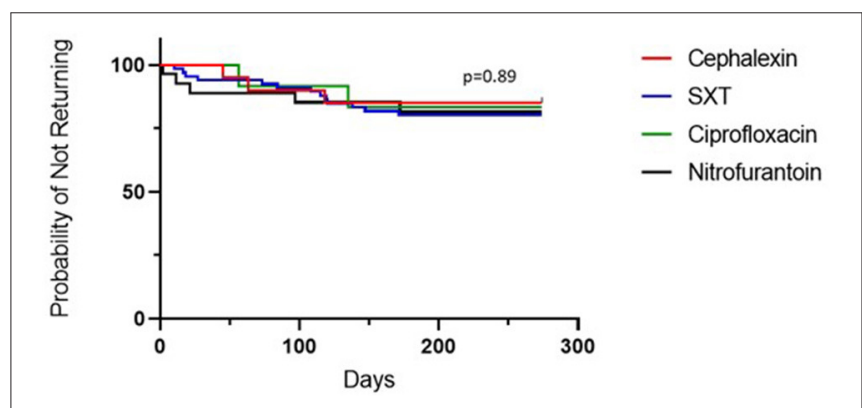

FIGURE 5 | Percentage of patients who did not return to the clinic for recurrent and/or persistent UTI symptoms in the 6 months following diagnosis, by antibiotic. Kaplan-Meier estimates for patients receiving each antibiotic.

antibiotic treatment did not occur. The latter is understandable since the treatment course is relatively short and changing a prescription would result in increased patient costs. Cephalexin was also associated with a low rate of symptom recurrence in this study compared to SXT or nitrofurantoin. Cephalexin may also improve adherence as it has a more favorable drug frequency (twice per day vs. four times per day with nitrofurantoin) $(5,14,15)$. Taken together, the results suggest that a firstgeneration cephalosporin might prove to be a better empiric first-line therapy for pediatric outpatient UTI compared to SXT, nitrofurantoin, or ciprofloxacin. 
The finding that providers preferentially selected SXT as the empiric antibiotic for UTI is similar to the adult literature, where SXT was empirically prescribed even when there were higher rates of resistance prevalent in the community $(16,17)$. The Infectious Diseases Society of America (IDSA) guidelines recommend that an antibiotic not be empirically prescribed if local resistance rates exceed $20 \%$ (6). The overall E. coli resistance rate to SXT was $20.2 \%$ in the current study. These findings highlight the need for outpatient antimicrobial stewardship interventions and education.

Antibiotics were discontinued in only three of the 48 (6.3\%) patients with negative urine cultures, which is consistent with an earlier study in a pediatric urgent care network which found that antibiotics were discontinued in only $4 \%$ of patients with negative urine cultures. The authors introduced a quality improvement educational intervention, which led to a greatly increased discontinuation rate of $84 \%$ (18). It is unclear what barriers may prevent providers from discontinuing antibiotics in the context of negative urine cultures and this area deserves more study, in order to design appropriate educational interventions and support mechanisms. Our findings suggest that quality improvement interventions designed both at discontinuing antibiotics when cultures are negative but also changing the choice of empiric front-line therapy (when appropriate) are needed.

It is important to point out that while E. coli was the most commonly identified pathogen in our study, as expected, the frequency $(50 \%)$ was lower than the 78 and $80 \%$ previously reported $(3,19,20)$. Our second most common microbiological result was mixed cultures, which could be attributed to suboptimal techniques used to collect urinary samples in the outpatient setting.

\section{REFERENCES}

1. Urinary Tract Infection: Clinical Practice Guideline for the Diagnosis and Management of the Initial UTI in Febrile Infants and Children 2 to 24 Months. Pediatrics. (2011) 128:595-610. doi: 10.1542/peds.2011-1330

2. Schmidt B, Copp HL. Work-up of pediatric urinary tract infection. Urol Clin. (2015) 42:519-26. doi: 10.1016/j.ucl.2015.05.011

3. Gorelick MH. Both gram stain and urine dipstick analysis were accurate in diagnosing urinary tract infection in children. Evidenced-Based Nurs. (2000) 3:86. doi: 10.1136/ebn.3.3.86

4. Harsh KM, Hetal NJ, Bhairavi MS. Urinary tract infection in children: clinical aspects and utility of urine dipstick test. Int J Contemp Pediatr. (2017) 4:790-5. doi: 10.18203/2349-3291.ijcp20171491

5. White B. Diagnosis and treatment of urinary tract infections in children. Am Fam Physician. (2011) 83:409-15.

6. Gupta K, Hooton TM, Naber KG, Wullt B, Colgan R, Miller LG, et al. International clinical practice guidelines for the treatment of acute uncomplicated cystitis and pyelonephritis in women: a 2010 update by the Infectious Diseases Society of America and the European Society for Microbiology and Infectious Diseases. Clin Infect Dis. (2011) 52:e10320. doi: $10.1093 / \mathrm{cid} /$ ciq257

7. Robinson JL, Finlay JC, Lang ME, Bortolussi R, Canadian Paediatric Society, Community Paediatrics Committee, Infectious Diseases and Immunization Committee. Urinary tract infection in infants and children: diagnosis and management. Paediatr Child Health. (2014) 19:315-9. doi: 10.1093/pch/19.6.315
Advantages to the current study include the large patient population including school-based clinics and the use of a single microbiology laboratory. Limitations include the retrospective nature, reliance on medical record documentation to identify patients, limited information regarding duration of antibiotic treatment, provider variability in use of rapid screening tests and potential variability in urine collection methods. It is possible that we did not capture all recurrences as patients may have sought care outside this healthcare system. In addition, the findings may not be generalizable to other communities where susceptibility patterns may differ. It is, however, very reassuring that in a large urban community, the majority of isolates were $E$. coli that were susceptible to cephalexin. Our findings suggest that there is a need for antibiotic stewardship interventions in the outpatient setting to promote the empiric use of narrow-spectrum antibiotics for pediatric patients with UTI symptom.

\section{DATA AVAILABILITY STATEMENT}

The raw data supporting the conclusions of this article will be made available by the authors, without undue reservation.

\section{AUTHOR CONTRIBUTIONS}

$\mathrm{BH}, \mathrm{VS}$, and PL were involved in the development of the concept and the design of the review, acquisition and gathering of data, statistical analysis, interpretation of results, and drafting and revising the manuscript. MK contributed to study design, reviewed the statistical analysis and performed additional analyses, and reviewed and edited the final manuscript. All authors approve the final manuscript as submitted.

8. Gupta K. Emerging antibiotic resistance in urinary tract pathogens. Infect Dis Clin North Am. (2003) 17:243-59. doi: 10.1016/S0891-5520(03)00006-0

9. Raz R, Chazan B, Kennes Y, Colodner R, Rottensterich E, Dan M, et al. Empiric use of trimethoprim-sulfamethoxazole (TMP-SMX) in the treatment of women with uncomplicated urinary tract infections, in a geographical area with a high prevalence of TMP-SMX-resistant uropathogens. Clin Infect Dis. (2002) 34:1165-9. doi: 10.1086/339812

10. Bryce A, Hay AD, Lane IF, Thornton HV, Wootton M, Costelloe C. Global prevalence of antibiotic resistance in paediatric urinary tract infections caused by Escherichia coli and association with routine use of antibiotics in primary care: systematic review and meta-analysis. BMJ. (2016) 352:i939. doi: 10.1136/bmj.i939

11. Arslan H, Azap ÖK, Ergönül Ö, Timurkaynak F. Risk factors for ciprofloxacin resistance among Escherichia coli strains isolated from community-acquired urinary tract infections in Turkey. J Antimicrob Chemother. (2005) 56:9148. doi: 10.1093/jac/dki344

12. Shaikh S, Fatima J, Shakil S, Rizvi SM, Kamal MA. Antibiotic resistance and extended spectrum beta-lactamases: types, epidemiology and treatment. Saudi J Biol Sci. (2015) 22:90-101. doi: 10.1016/j.sjbs.2014. 08.002

13. Clinical and Laboratory Standards Institute. Performance Standards for Antimicrobial Susceptibility Testing. Wayne, PA: CLSI supplement M100. (2015).

14. El-Rachidi S, Larochelle JM, Morgan JA. Pharmacists and pediatric medication adherence: bridging the gap. Hosp Pharm. (2017) 52:12431. doi: 10.1310/hpj5202-124 
15. Nitrofurantoin. In: Lexi-Drugs Online. Hudson (OH): Lexicomp, Inc. (2021). Available online at: http://online.lexi.com (accessed July 29, 2021).

16. Boggan JC, Navar-Boggan AM, Jhaveri R. Pediatric-specific antimicrobial susceptibility data and empiric antibiotic selection. Pediatrics. (2012) 130:e615-22. doi: 10.1542/peds.2012-0563

17. Copp HL, Shapiro DJ, Hersh AL. National ambulatory antibiotic prescribing patterns for pediatric urinary tract infection, 1998-2007. Pediatrics. (2011) 127:1027-33. doi: 10.1542/peds.2010-3465

18. Saha D, Patel J, Buckingham D, Thornton D, Barber T, Watson JR. Urine culture follow-up and antimicrobial stewardship in a pediatric urgent care network. Pediatrics. (2017) 139:e20162103. doi: 10.1542/peds.2016-2103

19. Conway PH, Cnaan A, Zaoutis T, Henry BV, Grundmeier RW, Keren R. Recurrent urinary tract infections in children: risk factors and association with prophylactic antimicrobials. JAMA. (2007) 298:17986. doi: 10.1001/jama.298.2.179

20. Paschke AA, Zaoutis T, Conway PH, Xie D, Keren R. Previous antimicrobial exposure is associated with drug-resistant urinary tract infections in children. Pediatrics. (2010) 125:664-72. doi: 10.1542/peds.2009-1527
Conflict of Interest: The authors declare that the research was conducted in the absence of any commercial or financial relationships that could be construed as a potential conflict of interest.

Publisher's Note: All claims expressed in this article are solely those of the authors and do not necessarily represent those of their affiliated organizations, or those of the publisher, the editors and the reviewers. Any product that may be evaluated in this article, or claim that may be made by its manufacturer, is not guaranteed or endorsed by the publisher.

Copyright $\odot 2021$ Lee, Kim, Herold and Soma. This is an open-access article distributed under the terms of the Creative Commons Attribution License (CC BY). The use, distribution or reproduction in other forums is permitted, provided the original author(s) and the copyright owner(s) are credited and that the original publication in this journal is cited, in accordance with accepted academic practice. No use, distribution or reproduction is permitted which does not comply with these terms. 